\title{
Translational Nano-Medicines: Targeted Therapeutic Delivery for Cancer and Inflammatory Diseases
}

\author{
Meghna Talekar, ${ }^{1}$ Thanh-Huyen Tran, ${ }^{1}$ and Mansoor Amiji ${ }^{1,2}$
}

Received 15 February 2015; accepted 10 April 2015; published online 29 April 2015

\begin{abstract}
With the advent of novel and personalized therapeutic approaches for cancer and inflammatory diseases, there is a growing demand for designing delivery systems that circumvent some of the limitation with the current therapeutic strategies. Nanoparticle-based delivery of drugs has provided means of overcoming some of these limitations by ensuring the drug payload is directed to the disease site and insuring reduced off-target activity. This review highlights the challenges posed by the solid tumor microenvironment and the systemic limitations for effective chemotherapy. It then assesses the basis of nanoparticle-based targeting to the tumor tissues, which helps to overcome some of the microenvironmental and systemic limitations to therapy. We have extensively focused on some of the tumor multidrug resistance mechanisms (e.g., hypoxia and aerobic glycolysis) that contribute to the development of multidrug resistance and how targeted nano-approaches can be adopted to overcome drug resistance. Finally, we assess the combinatorial approach and how this platform has been used to develop multifunctional delivery systems for cancer therapy. The review article also focuses on inflammatory diseases, the biological therapies available for its treatment, and the concept of macrophage repolarization for the treatment of inflammatory diseases.
\end{abstract}

KEY WORDS: aerobic glycolysis; hypoxia; inflammatory disease; macrophage repolarization; multidrug resistance; targeted biological therapies.

\section{INTRODUCTION}

Cancer is a disease where cells undergo transformation to obtain limitless replication, which enables them to proliferate aggressively into a mass of cells. It is a major public health concern with approximately 1.5 million cases being

\footnotetext{
${ }^{1}$ Department of Pharmaceutical Sciences, School of Pharmacy, Bouve College of Health Sciences, Northeastern University, Boston, Massachusetts 02115, USA.

${ }^{2}$ To whom correspondence should be addressed. (e-mail: m.amiji@neu.edu)
}

ABBREVIATIONS: 2DG，2-Deoxy-D-glucose; 2FDG，2Fluorodeoxyglucose; 5TDG, 5-Thio-D-glucose; AP-1, Activator protein-1; Arg, Arginase; asODN, Antisense surviving oligodeoxynucleotides; BAFF, B cell activating factor; BBB, Blood brain barrier; CAM, Cell adhesion molecules; CD44, Cluster of differentiation 44; CIA, Collagen-induced arthritis; EGFR, Epidermal growth factor receptor; EPR, Enhanced permeability and retention; FR, Folate receptor; GADPH, Glyceraldehyde 3phosphate dehydrogenase; GeRPs, Glucan-encapsulated siRNA particles; GLUT-1, Glucose transporter 1; GM-CSF, Granulocytemacrophage colony-stimulating factor; GM-BMM, GM-CSF derived bone marrow-derived macrophages; GTC, Galactosylated trimethyl chitosan-cysteine; HA, Hyaluronic acid; HA-AuNP/TCZ, Hyaluronate/gold nanoparticle/tocilizumab; HK2, Hexokinase-2; HIF, Hypoxia inducible factors; IBD, Inflammatory bowel disease; diagnosed in 2014 (1). Currently, surgery, chemotherapy, and radiation therapy continue to be the mainstay for the treatment of cancer. Recently, there has been a focus on developing more personalized cancer therapeutic options, which has led to the development of immunotherapy (2) and gene therapy (3). Despite the improvements in therapeutic

IL, Interleukin; iNOS, Inducible nitric oxide synthase; IRF, IFNregulatory factor; KLF4, Kruppel-like factor 4; LDHA, Lactate dehydrogenase A; LPS, Lipopolysaccharide; MTC, Mannose-modified trimethyl chitosan-cysteine; NiE, Nanoparticles-in-emulsions; PAMAM, Polyamidoamine; PBAE, Poly( $\beta$-amino ester); PCL, Poly(epsilon-caprolactone); pDNA, Plasmid DNA; PEI, Poly(ethylene imine); PEG, Polyethylene glycol; PEO-PCL, Poly(ethylene oxide)-poly(epsilon-caprolactone); PFK, Phosphofructokinase; PgP, P-glycoprotein; PKM2, Pyruvate kinase isoenzyme M2; PLGA, Poly(lactic-co-glycolic acid); PLK-1, Polo-like kinase; PPAR- $\gamma$, Peroxisome proliferator-activated receptor gamma; MDR, Multidrug resistance; miRNA, MicroRNA; NAT, Nucleic acid therapies; NF-кB, Nuclear factor kappa-light-chain-enhancer of activated B cells; NiE, Nanoparticles-in-emulsions; NiMOS, Nanoparticles-in-microspheres; OXPHOS, Oxidative phosphorylation; RA, Rheumatoid arthritis; RNase, Ribonuclease; RNAi, RNA interference; siRNA, Short interfering RNA; SLE, Systemic lupus erythematosus; STAT6, Signal transducer and activator of transcription 6; TNBS, 2,4,6Trinitrobenzenesulfonic acid; TNF- $\alpha$, Tumor necrosis factor alpha; UC, Ulcerative colitis; VACM-1, Vascular cell adhesion molecule-1; VEGF, Vascular endothelial growth factor. 
approaches, cancer treatments pose several limitations including poor pharmacokinetics properties, lack of water solubility, lower therapeutic indices, and development of drug resistance, which makes many of the novel approaches ineffective. Hence delivery strategies are actively being designed to overcome some of these limitations. Nanoparticle-based delivery systems have been pursued for the delivery of therapeutic agents in cancer and inflammation. Nanoparticles have diameters of less than $100 \mathrm{~nm}$ and vary in shape, size, and materials used for their preparation. In this review, we assess combinatorial nano-delivery strategies for the treatment of resistant cancer with an emphasis on the development of multifunctional nanoparticles for cancer therapy.

Inflammatory disease is another therapeutic area where the need for an optimal delivery system has been keenly pursued. Inflammation is a normal immune response, which occurs following exposure to infection or injury. Following resolution of the infection or injury, the inflammatory response subsides and allows the tissue to heal. Inability of the immune system to control the response leads to the development of an inflammatory disease. Several therapeutic strategies are available for the treatment of inflammatory disease $(4,5)$. In this review, we discuss the role of immune modulation using targets ranging from antigen-presenting cells to activated $\mathrm{T}$ cells, macrophages, and $\mathrm{B}$ cells for the treatment of inflammatory diseases. Further to this, we also assess the use of biological therapy for inflammatory disease and discuss the potential delivery challenges. Finally, we address the concept of macrophage repolarization strategy for inflammatory disease with a focus on macrophage polarization spectrum, repolarization as a strategy for antiinflammatory therapy, and macrophage-targeted delivery systems.

\section{CANCER THERAPY AND DRUG DELIVERY CHAL LENGES}

\section{Solid Tumor and the Microenvironment}

Solid tumors consist of cancer cells and stromal cells (including fibroblasts and inflammatory cells) embedded in an extracellular matrix and nourished with a vascular network $(6,7)$. The communication between these cells modulated by an alteration in the synthesis of growth factors, chemokines, and adhesion molecules affects the malignant transformation of cancer cells making them more susceptible to metastasize. Likewise, the tumor stroma reduces the difference in the arteriole and venule pressure and increases the geometric resistance preventing drug delivery within the tumor tissues (8). Similarly, the aberrantly developed tumor vasculature also prevents consistent delivery of drugs to the tumor tissue leading to the development of drug resistance (9). Like the tumor vasculature, solid tumors often lack or have fewer functional lymph vessels than normal tissues. This contributes to an increase in interstitial fluid pressure, which inhibits the distribution of larger molecules via convection and further compresses the blood vessels diverting the blood flow from the tumor core to the periphery. Thus the poorly developed vasculature, lack of efficient lymphatic drainage, impaired tumor blood flow, vascular compression by surrounding cancer cells, and development of hypoxic regions limit exposure and penetration of cytotoxic drugs to the tumor tissue.

\section{Systemic Chemotherapy Delivery Challenges}

Despite the solid tumor microenvironmental factors hindering the delivery of cytotoxic agents to the tumor tissue, chemotherapy continues to be used as a mainstay for the treatment of cancer. Following its administration, several systemic delivery challenges are encountered including inadequate oral absorption, increased first pass, or rapid clearance. Apart from the pharmacokinetic challenges, other factors within the tumor microenvironment can contribute to increased drug resistance. Hydrophobic cytotoxic drugs are substrates for ATP-dependent efflux pumps due to which they often show lower intracellular concentrations (10). Similarly, some cytotoxic agents show reduced drug uptake thus exhibiting increased resistance. Apart for modulating intracellular drug uptake, cancer cells often regulate their endogenous detoxification mechanisms (e.g., CYP450 enzymes) to enhance drug clearance thus increasing drug resistance. Development of drug resistance by these mechanisms also leads to the development of cross-resistance to other unrelated drugs inducing the development of multidrug resistance (MDR).

Apart from the pharmacokinetic and MDR-based challenges to chemotherapy, several on-target and off-target toxicities are often observed with chemotherapeutic agents (11). On-target toxicity includes development of adverse effects due to action of the therapeutic agent on the desired site including development of a rash or hypertension. These on-target toxicities are difficult to avoid without affecting drug potency. On the other hand, off-target toxicity is observed due to inhibition or effect on unintended sites. Off-target toxicity can often be overcome by structural design modulations. Conventional cytotoxic agents such as paclitaxel, doxorubicin, and etoposide often exhibit off-target effects. Although targeted therapeutic strategies have been designed, they are not completely devoid of adverse effects opening the possibility of designing targeted delivery alternatives.

In search for reducing off-target effects and achieving personalized anticancer therapy, focus has been on developing nucleic acid therapies (NAT). Nucleic acids are hydrophilic, highly negatively charged molecules which enable sequence-specific targeting of mRNAs thus modulating gene expression. Short interfering RNA (siRNA) and microRNA (miRNA) are NATs which had been investigated for cancer therapy. Naked delivery of these NATs has been limited for localized ocular therapy (Vitravene $\AA$ for the treatment of cytomegalovirus (12), anti-vascular endothelial growth factor (VEGF) inhibitor (Macugen $\AA$ ) for the treatment of agerelated macular degeneration (13)) and infectious respiratory disease. Non-carrier-based systemic delivery of NAT is limited due to poor ribonuclease (RNase) resistance, which yields a very short half-life (2-6 min), inability to bind strongly to plasma proteins which limits tissue accumulation, and rapid clearance through the kidneys due to their small size. Several nanoparticle-based carrier systems have been designed to overcome some of these barriers associated with the delivery of NATs. 


\section{Passive Tumor Targeting}

The basis of delivery of nanoparticles to tumor tissue is broadly classified as passive and active targeting. Passive targeting of nanoparticles depends on enhanced permeability and retention (EPR) effect, characterized by greater tumor accumulation of nanoparticles due to leaky tumor vasculature and inefficient lymphatic drainage. The EPR effect is influenced by the size and the surface charge of the nanoparticle system Nanocarriers in the size range of 10 $100 \mathrm{~nm}$ and either slightly positive or negatively charged typically show improved accumulation at the tumor site after prolonged circulation (14). The nanoparticles are also surface coated with polyethylene glycol (PEG) to enhance systemic circulation. Inclusion of PEG hinders protein adsorption and recognition by the phagocytic system improving systemic circulation time. Although passive targeting targets nanoparticles to the tumor tissue, a majority of the dose does not extravasate to the tumor tissue due to capillary disorder, blood flow, and rate of lymphatic drainage, which tend to vary between and within tumor tissues. Extravasation and accumulation also does not mean that successful therapy can be attained, as the therapeutic payload has to reach the intended cell, cytosol, or organelle for effective activity.

\section{Active Tumor Targeting}

Active targeting of nanoparticles is an approach that helps to target the nanocarriers specifically to the intended site post-delivery into the tumor microenvironment. It is attained by coating the surface of the particles with a ligand that recognizes a target within the tumor tissue. For successful targeting, it is critical to use a target which has tumor specificity, has homogenous expression, and is not shed or downregulated. The ligand should have stability over the processing conditions posed during the nanoparticle preparation process. After administration, the ligand should be cleared from the systemic circulation and it should not induce an immune reaction. The nanoparticle surface should have optimal ligand functionalization to enable binding at multiple sites on the tumor site. Post binding the ligand should be internalized and the attached payload should be released quickly and relatively easily. Following delivery of the drug payload, the ligand should be metabolized and eliminated without toxicity.

\section{Types of Targeting Moiety}

Targeting of nanoparticles can be achieved by using various targeting ligands including antibodies, peptides, nucleic acid aptamers, carbohydrates, and small molecules. Use of these targeting ligands provides tumor-specific delivery with reduced localization to the liver and spleen. Despite the use of targeting ligands, an optimal balance between the targeting ligand and PEG is critical to achieve effective targeting while maintaining PEG-mediated stealth effect preventing premature clearance. Some of the popular receptors of cancer cells, which have been investigated for nanoparticle targeting, include medium-sized molecules (folate $(15,16)$, galactose (17), aptamers (Pegaptanib) (18), proteins and peptides (transferrin) (19), and epidermal growth factor peptide (15) and antibodies (Herceptin (Trastuzumab) (20), Rituxan (Rituximab)) (21). In our laboratory, we have developed several EGFR-targeted particles for pancreatic (22,23), breast (Fig. 1) (24), and ovarian cancer (15) including several multidrug-resistant cancers.

\section{OVERCOMING TUMOR MULTIDRUG RESISTANCE}

\section{Tumor Hypoxia, Aerobic Glycolysis, and MDR}

Hypoxia ( $\leq 2 \%$ oxygen) or severe hypoxia/anoxia ( $\leq 0.02 \%$ oxygen) is the reduction of oxygen levels known to occur with various pathological conditions including development of solid tumors $(25,26)$. In solid tumors, the vascular heterogeneity observed with an increase in tumor size leads to inconsistent distribution of nutrients and oxygen generating hypoxic areas in the tumor tissue. Presence of these hypoxic microenvironments is a poor prognostic indicator due to its effect on drug resistance and malignant phenotype (Fig. 1) (27). In hypoxia, drug resistance and malignancy can be attained by direct mechanisms (by overproduction of nucleophilic substances, decreased generation of free radicals, and increased production of free radical scavengers) or indirect mechanisms (pre-DNA-synthetic arrest, upregulated DNA repair enzyme activity, overproduction of metallothioneins (28). Hypoxia inducible factors (HIFs) are DNA-binding transcription factors which associate with specific nuclear cofactors to activate genes that enable cancer cells to adapt to compromising condition posed in hypoxia (29). Upregulation of HIF1- $\alpha$ system at the cellular level induces a multiphasic response, which affect genes involved in the glycolytic pathway that promote tumor angiogenesis, proliferation, and metastasis. Clinically both HIF- $1 \alpha$ and HIF- $2 \alpha$ are upregulated in a variety of human tumors including bladder, breast, colon, glial, hepatocellular, ovarian, pancreatic, prostate, and renal tumors and their elevated expression is correlated with poor patient outcome (30). Experimental alterations of HIF-1 $\alpha$ expression has shown an increase in tumor growth and a loss of HIF activity has been shown reduced tumor growth, vascularization, and energy metabolism (31).

Although these mechanisms offer cancer cells a survival advantage from a metabolic perspective, the fluctuating oxygen levels in hypoxia would prove lethal to cancer cells if oxidative phosphorylation (OXPHOS) was used as a primary source of ATP generation. Hence cancer cells are programmed to adapt to glycolysis as a major mechanism for generating ATP. Apart from the role of glycolysis in hypoxia, cancer cells depend on glycolysis as a primary source of ATP generation even under aerobic conditions a phenomenon termed as aerobic glycolysis. Aerobic glycolysis offers cancer cells several growth and drug resistance advantages. Generation of bicarbonic and lactic acid as end products of glycolysis allows cancer cells to sustain a tumor microenvironment that promotes tumor invasion and suppresses anticancer immune effectors providing radio and chemotherapeutic resistance (32). From a therapeutic perspective, effect of hypoxia and aerobic glycolysis in the survival of cancer cells offers possibilities of developing therapeutic strategies that affect key processes in these pathways providing promising anticancer therapy alternatives. 

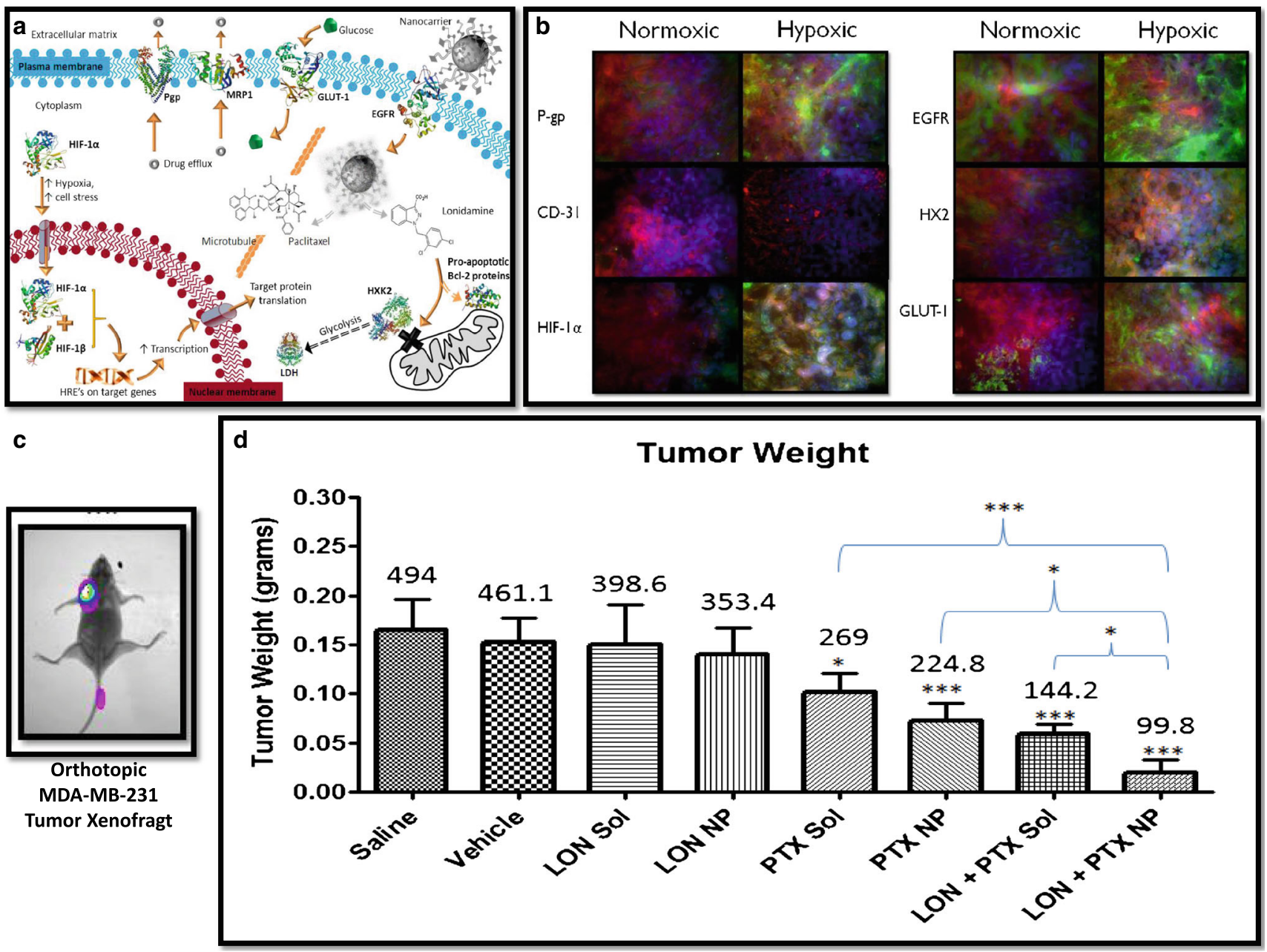

Fig. 1. a Tumor MDR mechanisms including hypoxia, aerobic glycolysis, and strategies to overcome resistance using targeted nanoparticlebased delivery. b MDA-MB-231 estrogen-negative human breast tumor xenograft was established orthotopically in the mammary fat pad of female athymic $(\mathrm{nu} / \mathrm{nu})$ mice using cells cultured under normoxic and hypoxic conditions. At the time of excision, the tumor size was the same $\sim 100 \mathrm{~mm}^{3}$. c EGFR-targeted nanoparticles showing preferential accumulation in tumor tissue and $\mathbf{d}$ the mean weight and volumes of tumors upon treatment with different formulations on day 28 drug post-administration. The dose of paclitaxel (PTX) was $20 \mathrm{mg} / \mathrm{kg}$ and lonidamine (LON) was $80 \mathrm{mg} / \mathrm{kg}$ administered in MDA-MB-231 human breast cancer-bearing female athymic (nu/nu) mice. Reprinted from Milane et al., Therapeutic Efficacy and Safety of Paclitaxel/Lonidamine Loaded EGFR-Targeted Nanoparticles for the Treatment of Multidrug-Resistant Cancer. Plos One. (2011). DOI: 10.1371/journal.pone.0024075

\section{Overcoming MDR by Effect on Hypoxia and Inhibition of Glycolysis}

Treatment of hypoxic conditions has been reported by two independent chemotherapeutic routes; first is use of bioreductive prodrugs that are selectively activated in the hypoxic environment and secondly small molecule drugs against signature molecular targets of hypoxia. Bioreductive prodrugs are derivatives of five chemical groups (nitro groups, quinones, aliphatic, or aromatic N-oxides, and transition metals) that are enzymatically activated under reducing environment to their active forms. Typically the non-toxic prodrug is enzymatically converted to free radical form, which forms superoxide under normoxia regenerating the original prodrug. Under hypoxic conditions, the free radical undergoes further reaction to achieve a cytotoxic form. Several bioreductive drugs under preclinical and clinical development have been discussed comprehensively in a recent review (26). Among molecular targets, HIF-1 $\alpha$ has been the most extensively studied in hypoxia primarily due to its involvement with several hypoxia related as well as unrelated molecular pathways. Several chemodrug have understandably been developed to block HIF-1 $\alpha$ expression, transcription, translation, target-binding disruption, and other associated signaling molecules. With the advent of novel nanoparticles, several strategies have been reported targeting hypoxia (33). Thambi et al., have developed a hypoxiaresponsive nanoparticle using 2-nitroimidazole derivative of carboxymethyl dextran that efficiently delivered the drug payload to the tumor under hypoxic conditions resulting in enhanced antitumor activity relative to control animals treated with saline (34). Yet another report developed $\mathrm{pH}$ responsive nanoparticles that specifically targeted tumor in vivo and shed its protective coating when internalized into the hypoxia environment leading to site-specific payload delivery (35).

siRNA loaded in block copolymer nanoparticles have also been successfully delivered to tumors under hypoxic 
environment resulting in silencing the expression of HIF-1 $\alpha$ gene, thereby increasing the antitumor efficacy of doxorubicin drug in prostate cancer xenograft model (36). Similarly, antisense oligonucleotide inhibition of HIF-1a has shown to prevent upregulation of $\mathrm{P}$-glycoprotein $(\mathrm{PgP})$ indicating hypoxia induced $\mathrm{PgP}$ is via $\mathrm{HRE}$ on MDR-1. RNA interference (RNAi)-based inhibition of HIF-1a RNA has shown a reversal in hypoxia induced cisplatin and doxorubicin resistance. Though these recent reports exhibit promising therapeutic efficacy, the true potential of such nanoparticle in targeting hypoxia are yet to be fully explored.

Similar to HIF-1 $\alpha$ downregulation, inhibition of glycolysis as a therapeutic strategy has been avidly explored for resistance cancer. Glucose transporter 1 (GLUT1), hexokinase-2 (HK2), glyceraldehyde 3-phosphate dehydrogenase (GADPH), phosphofructokinase (PFK), pyruvate kinase isoenzyme M2 (PKM2), and lactate dehydrogenase A (LDHA) are the popular molecular targets for development of metabolic inhibitors, though other enzymes have also been explored. Small molecules such as fasentin, WZB117, and STF-31 have been assessed as prominent inhibitors of GLUT1. Specific targeting of glucose transporters in cancer cells would be a challenge and hence glucose transporters are now being investigated as targeting receptors to ferry drug-loaded nanoparticles inside the cells as also across blood-brain barrier (BBB) (37). Glucose analogues such as 5-thio-D-glucose (5TDG), 2-deoxy-D-glucose (2DG), and 2-fluorodeoxyglucose (2FDG) have also been reported for effective anticancer therapy. 2DG has also been associated with abnormal GlcNAcylation of proteins causing ER stress and rendering cancer cells more sensitive to other anticancer drugs (38). 3-Bromopyruvate and lonidamine are direct inhibitors of HK enzyme and are currently studied for preclinical and clinical application. In our laboratory, we have investigated development of EGFR-targeted nanoparticles delivery of these particles. Combination of paclitaxel and lonidamine has shown favorable biodistribution and significant tumor growth suppression with nonspecific toxicity compared to individual drug-loaded nanoparticles as well as combination dosed in solution in hypoxia conditioned breast cancer in vivo $(24,39)$. Other enzymes involved in glycolysis have also been studied for curbing anticancer therapy and most of these are in clinical or preclinical stage of development. Among these PKM-2 has been studied extensively due to its overexpression in tumors. Novel inhibitors and nucleic acid therapies have been designed to target PKM-2 to achieve the desired anticancer activity $(40,41)$. Goldberg et al., screened a library of siPKM-2 and identified a specific PKM-2 siRNA (si156) which when encapsulated in lipidoid nanoparticles showed tumor regression in HepG2 and SKOV3 xenograft tumor models by inducing cell apoptosis (42). We have also assessed the combination of siPKM-2 with siMDR-1 and paclitaxel and have observed significant improvement in antitumor efficacy especially in drug-resistant ovarian cancer (unpublished). Thus nano-delivery systems offer a viable and effective therapeutic strategy to revisit some potent inhibitors of glycolytic pathway or enhance delivery of novel therapeutic options for the treatment of resistant cancers.

\section{Overcoming MDR by Modulating Apoptotic Genes and Proteins}

ATP mediated efflux pumps is one of the most well studied mechanism of MDR and several therapeutic strategies using small molecules and biologics have been used to overcome this resistance $(43,44)$. However drug resistance attributed to activation of antiapoptotic cellular defense is also known to lead to drug resistance. Thus modulation of antiapoptotic genes provides another optimal strategy for overcoming MDR in tumor cells that do not rely on efflux pumps for resistance. Cisplatin is a chemotherapeutic agent known to induce cell death by activation of caspase that cleaves cellular proteins and subsequently induces apoptosis. Survivin and bcl-2 are two antiapoptotic genes overexpressed in non-small cell lung cancer. Overexpression of bcl-2 is known to delay the onset of apoptosis induced by several chemotherapeutic drugs. Evidence reported indicates that downregulation of antiapoptotic genes such as survivin and bcl-2 can sensitize cancer cells to anticancer drugs $(45,46)$. We have previously observed the effect of combining siRNA and cisplatin in CD44targeting hyaluronic acid (HA)-based self-assembling nanosystems reversed drug resistance and delayed the tumor growth significantly (growth inhibition increased from 30 to $60 \%$ ) in cisplatin-resistant tumors without indication of non-specific toxicity (Fig. 2).

An alternative strategy of survivin knockdown was assessed by delivering antisense survivin oligodeoxynucleotides (asODN) in polyamidoamine (PAMAM) dendrimer modified magnetic nanoparticles. The PAMAM component of these particles protected the asODN from degradation while the magnetic component enhanced binding, uptake, and provided an imaging modality (47). In comparison to nanoparticles conjugated with nonsense (non-complementary to survivin mRNA) these particles silenced survivin mRNA and protein expression.

Modulation of MDR by altering cellular apoptotic mechanism has also been achieved by the coadministration of ceramide. Ceramide is a signaling agent that affects differentiation, proliferation, immune response, and apoptosis. It is known to affect cellular apoptosis by activating several apoptotic inducers and also by increasing mitochondrial outer membrane permeability that allows the passage of pro-apoptotic protein release. We have previously investigated polymeric nanoparticle formulation of poly(ethylene oxide)-poly(epsilon-caprolactone) (PEO-PCL) to coadminister ceramide with paclitaxel in MDR human ovarian cancer cell lines (48). Combination treatment paclitaxel and ceramide loaded PEO-PCL nanoparticles increased the cytotoxicity of paclitaxel. Similarly, EGFRtargeted polymeric nanoparticles when delivered in combination with paclitaxel and lonidamine in an orthotopic MDR human breast cancer model showed tumor accumulation within 3 to $4 \mathrm{~h}$, a 5-fold reduced tumor volume compared to control (Fig. 1) and altered the MDR phenotype of the tumor xenografts. Thus these novel delivery systems can be used in combination with conventional therapy to modulate apoptotic pathways and achieve enhanced anticancer therapy.

\section{Overcoming MDR by Silencing Cell Cycle Checkpoint Genes}

RNAi-based therapeutic approach has also been adopted for silencing several cell cycle checkpoint genes. Polo-like kinase (Plk-1) is a mitotic cyclin-independent serine-threonine kinase involved in several cell cycle processes. It is known to be involved in centrosome separation and 
a

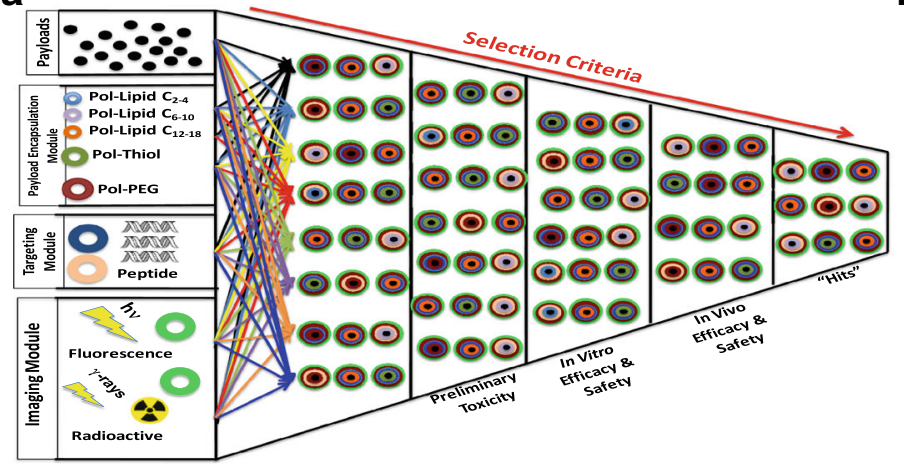

C

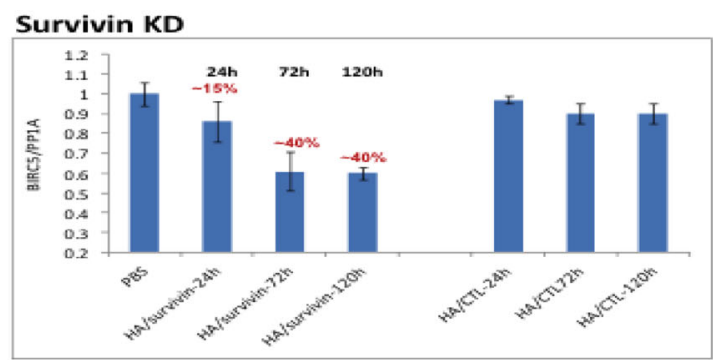

Bcl-2 KD

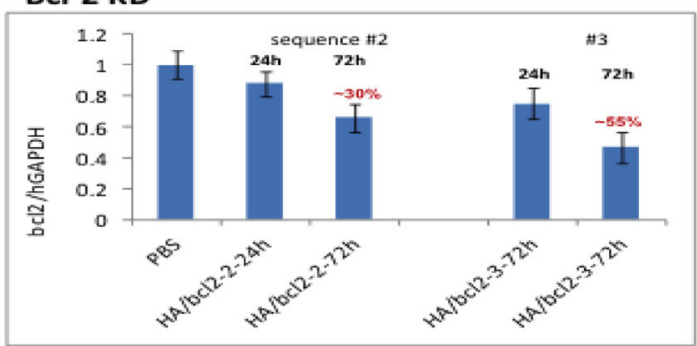

b

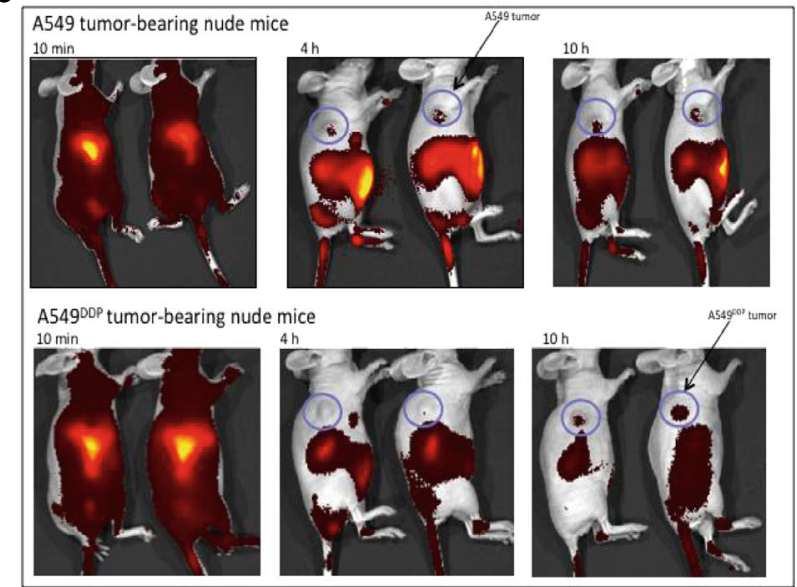

d

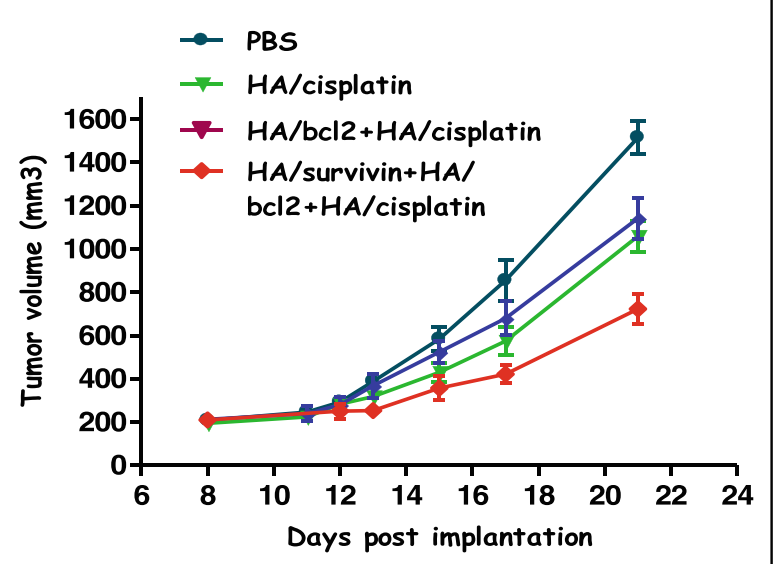

Fig. 2. a Combinatorial-designed nano-assemblies enabling selection of delivery components to achieve optimal encapsulation efficiency, minimal toxicity, and desired in vitro and in vivo activity. b Qualitative biodistribution analysis of indocyanine green encapsulated HA-PEI/HAPEG nanoparticles in human non-small cell lung cancer A549 and A549DDP (a) and small cell lung cancer H69 and H69AR cells (b) tumor bearing mice. Free indocyanine green dye was injected as control to see the clearance of dye from the circulation in mice (c). The images have been acquired using IVIS live imaging station. Reprinted from Ganesh et al., 2013 (c) In vivo Survivin and Bcl-2 Gene Silencing Efficacy in Mice Bearing A549 ${ }^{\text {DDP }}$ Cisplatin-Resistant NSCLC Xenografts (d) In vivo antitumor efficacy following combination Gene Silencing and Cisplatin Efficacy in Mice Bearing A549 ${ }^{\text {DDP }}$ NSCLC Xenografts

maturation and is known to be a critical regulator of mitotic progression in mammalian cells. Its expression is upregulated in pancreatic tumor tissue and it is involved in a wide variety of cell cycle processes. RNAi mediated depletion of Plk-1 has been investigated to assess the effect of sensitizing pancreatic tumor cells to gemcitabine. Yu et al., observed that Plk-1 overexpression was associated with reduced sensitivity to gemcitabine in pancreatic cells (49). However treatment with Plk-1 siRNA with subsequent treatment with gemcitabine dramatically downregulated Plk-1 expression and increased gemcitabine cytotoxicity in pancreatic tumor cells. Similar effect of siRNA mediated Plk-1 downregulation was observed in human prostate cancer (50) and human esophageal cancer cells (51).

In our laboratory, we also investigated silencing of Mad2, an essential mitotic checkpoint component required for accurate chromosome segregation during mitosis. Complete abolition of $\operatorname{mad} 2$ is known to lead to cell death and hence we developed an EGFR-targeted chitosan system for silencing the Mad2 gene in human A549 non-small cell lung cancer cells. These delivery systems showed time-dependent and selective intracellular internalization of EGFR-targeted nanoparticles compared to non-targeted system with a 5-fold greater accumulation with the targeted system within 15 min compared to non-targeted nanoparticles. The targeted nanoparticles showed nearly complete depletion of Mad2 expression in A549 cells contrasting with the partial depletion in the non-targeted system. Accordingly, Mad2-silencing-induced apoptotic cell death was confirmed by cytotoxicity assay and flow cytometry thus indicating the potential for selective and effective killing of cancer cells by inhibition of checkpoint genes (52).

\section{COMBINATORIAL-DESIGNED NANOSYSTEMS FOR COMBINATION THERAPY}

Polymeric-based delivery systems have been investigated for delivery of small molecules and biologics. Assessment of 
an individual polymer at a time is a time consuming approach, which requires a large number of resources for optimization. This has given rise to a need for comprehensive and highly flexible platforms that enable selection of promising candidates from a library of compounds to deliver small molecules, nucleic acids, and proteins.

\section{Cytotoxic Small Molecules}

Dextran has been studied as a delivery system using the combinatorial approach due to its high biocompatibility, biodegradability, and non-toxicity. The hydroxyl groups on the polymer backbone make it an ideal candidate for preparing polymeric nanoparticles using the combinatorial approach. Application of click synthesis chemistry enabled the preparation of O-pentynyl dextran, which could be used as a precursor to yield lipid, thiol, or PEG derivatives of dextran (53). In an aqueous environment the lipid derivatives self-assembled to form drug encapsulated nanoparticles. In these particles, shorter lipid chains showed better encapsulation of hydrophilic drugs whereas longer lipid chain modified dextran particles enhanced encapsulation of hydrophobic drugs. The stability of these particles was enhanced by thiol modifications whereas stability and stealth properties were enhanced by preparation of PEG dextran.

\section{Oligonucleotides (siRNA/microRNA)}

Langer lab previously reported parallel synthesis and combinatorial design of polymeric material where 7 different diacrylate monomers were modified with 20 different amine monomers to provide a library of 140 poly(b-amino ester) (PBAE) which were assessed for aqueous solubility and subsequent DNA complexing capability (54). Anderson et al. subsequently demonstrated the ability of these PBAE derivatives to be photocrosslinked to manipulate degradation behavior (55). Greenland et al. then assessed some of these PBAE candidates and showed improved in vivo transfection efficiency of plasmid DNA adjuvants for vaccine application (56). Poly[(1,5di(acryloxyethoxy)hexane)-co-(4- aminobutanol)] showed a seven-fold increase in gene expression and a $70 \%$ enhancement in subsequent immune response. This approach was extended further to develop a large library of lipid-like materials called "lipidoids" which used alkyl acrylate (or acrylamides), primary or secondary amines and these were screened for transfection efficiency.

Kobayashi et al. extended the application of dextran nanoparticles for the delivery of siRNA by loading MDR1 siRNA in octylamine-modified dextran, dextran-PEG, and dextran thiol (44). The nanoparticles were in the size range of $101 \pm 3 \mathrm{~nm}$ with a zeta potential of $-0.22 \mathrm{mV}$. In vitro MTT assay in drug-sensitive osteosarcoma and ovarian cancer cells (KHOS and SKOV3, respectively) showed significantly higher (5-10 fold) dose-dependent antiproliferative activity compared to free drug in solution and in a liposomal formulation. The improved toxicity was attributed to better drug delivery characteristics with the dextran particles. In drug-resistant cell lines, increase in resistance to free drug treatment and drug loaded in the liposomal formulations was observed. Similarly, nucleic acid transfection efficiency of fluorescently labeled MDR1 siRNA demonstrated uptake of the dextran nanoparticles within $2 \mathrm{~h}$ in drug-resistant KHOSR2 osteosarcoma cells. The enhanced uptake was translated into significant knockdown of $\mathrm{PgP}$ expression at a protein level confirming successful delivery of siRNA at its intended site.

HA like dextran is another natural polysaccharide that has been explored for designing a library of unique polymers using the combinatorial approach (Fig. 2). Due to its biodegradable, non-toxic, non-immunogenic, non-inflammatory properties of $\mathrm{HA}$, its use as a delivery system has been pursued with interest. In our laboratory, we have reported development of a library of amine derivatives of HA using EDC/NHS coupling chemistry. The activated backbone of HA can also be functionalized with primary monofunctional, bifunctional fatty amines and polyamines such as poly(ethylene imine) (PEI) and poly(L-lysine). Simultaneously, PEG can also be conjugated to attain surface stabilization and provide stealth effect to the nanoparticles on systemic administration. We have successfully assessed the formation of self-assembling nanoparticles using the HA-PEI/ HA-PEG system. In vitro assessment of these nanoparticles has shown nearly $55 \%$ gene silencing, conforming efficient intracellular delivery of the siRNA payload (46). In vivo biodistribution studies also showed enhanced tumor accumulation (Fig. 2) and $55 \%$ SSB downregulation. The effectiveness of these HA-PEI/ HA-PEG-based delivery system in vivo showed nearly $62 \%$ tumor suppression relative to other treatment groups (45) (Fig. 2). Thus our results with HA-PEI/HA-PEG systems clearly indicates development of a CD44-targeted delivery systems which can be modulated to enhance binding to cancer cells thus improving the performance of the existing drugs as well as drug delivery systems.

\section{INFLAMMATORY DISEASES AND BIOLOGICAL THERAPIES}

\section{Acute and Chronic Inflammation}

Inflammation is the immune response of the body against infection or injury. Acute inflammation has relatively short duration (2-3 days) with clinical signs including redness, heat, pain, and swelling at the site of injury due to the local responses of immune, vascular, and parenchymal cells (57). Upon infection or injury, inflammatory responses initiate by rapid neutrophil invasion due to increased permeability of blood vessel in the injury tissue, followed by recruitment of inflammatory cells such as macrophages, dendritic cells, and fibroblast. This initial inflammatory response becomes amplified by the overproduction of cytokines and chemokines by the inflammatory cells (58). The production of antiinflammatory cytokines such as interleukin-10 (IL-10) by the inflammatory cells is then to clear exogenous pathogens, inflammatory debris, and normalization of tissue structure and functions (57). However, if the inflammatory response is deregulated or initial stimulus causing inflammation is not eradicated, the acute inflammation can progress to chronic situation.

Chronic inflammation is a long-term reaction to an inflammatory stimulus which may last for weeks, months, or years, resulting in tissue damage in various chronic inflammatory diseases such as autoimmune diseases, metabolic disorders such as atherosclerosis and obesity, fibrosis, and cancer (59). In chronic inflammation, monocyte-derived 
macrophages are continuously recruited to the injury tissues and release high amount of pro-inflammatory mediators such as tumor necrosis factor alpha (TNF- $\alpha)$, interleukin-1-beta (IL-1 $\beta$ ), and prostaglandin that sustain the inflammatory response. Chronic inflammation has been divided into autoimmune diseases and autoinflammatory diseases (60). Autoreactive $\mathrm{T}$ cells are the main player to induce autoimmune diseases such as inflammatory bowel disease, rheumatoid arthritis, type I diabetes, psoriasis, lupus, and multiple sclerosis. In these diseases, autoreactive $\mathrm{T}$ cells are expanded and cannot be controlled by Treg cells. Figure 3 illustrates an example of disease progression in rheumatoid arthritis. Various cytokines such as TNF- $\alpha$, IFN- $\gamma$, IL-2, IL-12, IL-23, and IL-17 involve in maintaining autoreactive T cells. On the contrary, autoinflammatory diseases such as sepsis, gout and type II diabetes are mediated predominantly by cytokines of dysfunctional macrophages, particularly IL-1 $\beta$ (60).

\section{Biological Therapy for Inflammatory Diseases}

Biological therapy for inflammatory diseases involves using biological agents such as antibodies and nucleic acids to upregulate anti-inflammatory cytokines or selectively downregulate the inflammatory mediators.

\section{Downregulation of Inflammatory Cytokines}

TNF- $\alpha$ is mainly produced by macrophages and T cells and plays a key role in the pathogenesis of inflammatory bowel disease (IBD), rheumatoid arthritis (RA), and other autoimmune diseases (5). In IBD, TNF- $\alpha$ increases pro-inflammatory cytokine production, causes barrier alterations, and promotes cell death of intestinal epithelial cells and Paneth cells (4). In the rheumatoid joints, TNF$\alpha$ promotes chemokine production, and expression of cell adhesion molecules (CAMs) on endothelial cells, facilitating the migration of

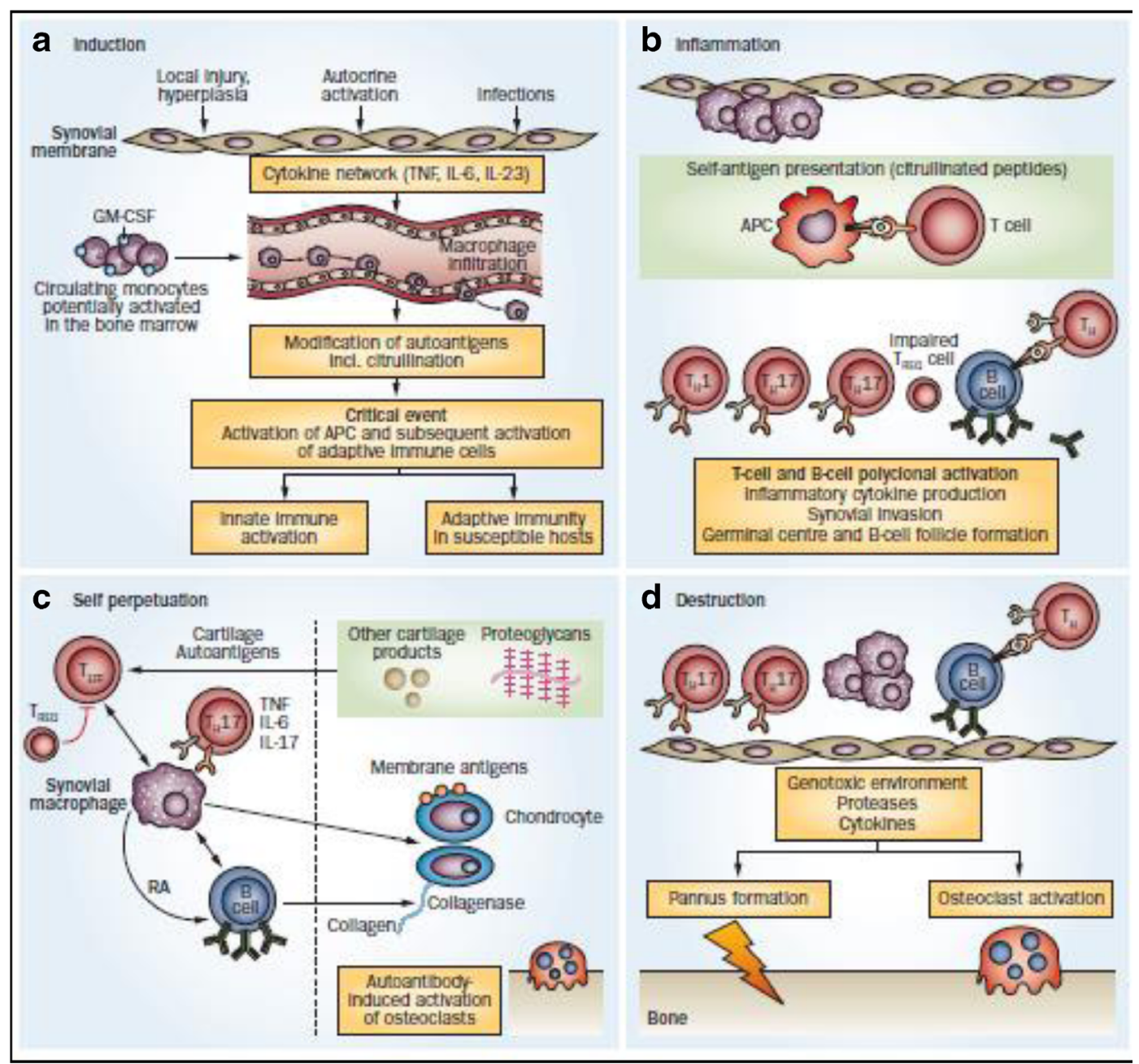

Fig. 3. Disease progression in rheumatoid arthritis (RA). a Induction phase: initial activation of the immune system leads to an inflammatory cascade. $\mathbf{b}$ Inflammation phase: self-antigen presentation leads to activation of $\mathrm{T}$ cells and B cells. c Self-perpetuation: cartilage autoantigens activate the immune system against cartilage tissue with infiltration of pannus into the joints resulting in tissue destruction. d Destruction phase: synovial fibroblasts and osteoclasts are activated by pro-inflammatory cytokines such as TNF and IL-6. Abbreviations: APC, antigenpresenting cell; GM-CSF, granulocyte-macrophage colony-stimulating factor; $T E F F$, effector T cell; $T H$, helper T (cell); TH1, type 1 helper T cell; TH17, type 17 helper T cell; TREG, regulatory T cell. Reprinted from Burmester et al., 2014 with permission from NPG 
inflammatory cells from the circulation into the synovium. Neutralization of TNF- $\alpha$ with antibodies such as infliximab and adalimumab has been shown to suppress experimental colitis and in patients with ulcerative colitis (UC) in both induction and maintenance therapy (61).

In addition to TNF- $\alpha$, IL- 6 is a cytokine with multifunctions in the pathogenesis of autoimmune diseases. In RA, IL6 induces join and cartilage destruction by stimulation of synoviocyte cell proliferation and osteoclast activation, and increasing production of MMPs together with IL-1 (62). IL-6 exerts its pro-inflammatory functions by binding to its receptor (IL-6R); the IL-6/IL-6R acts alone or may bind to glycoprotein 130 (gp 130) on the membrane to mediate signaling. Therefore, blockage of IL-6 activity may be achieved by reducing IL- 6 production, blocking IL- 6 binding to its receptor, and blocking the binding of IL-6/IL-6R complex to gp 130. Tocilizumab is the first monoclonal antibody against IL-6 signaling by blocking the interaction of IL-6 with IL-6R which has been shown to be effective for the treatment of RA, CD, and SLE (63).

Recently, IL-17 has been shown to contribute to the pathogenesis of IBD and RA. IL-17 acts on various cell types express IL-17 receptor (IL-17R) on their membrane such as immune cells, epithelia cells, and fibroblasts (64). IL-17 stimulates the production of pro-inflammatory cytokines TNF- $\alpha$, IL- 6 and IL$1 \beta$, and chemokines, promoting the recruitment and activation of neutrophils, lymphocytes, and macrophages, leading to local inflammation and tissue damages. IL-17 can also induce tissue injury by upregulating the expression of MMPs. The human antiIL17 antibody secukinumab is now entering phase III studies and shows to be effective in patients with RA (64).

More recently, IL-12 and IL-23 secreted by macrophages and dendritic cells have been recognized to involve in the pathogenesis of IBD by promotion of $\mathrm{T}$ cell differentiation to Th1 and Th17 cells which produce IL-17 cytokine. IL-12 and IL-23 share a common p40 subunit; therefore blocking the p40 subunit may inhibit both pathways. Neutralizing IL-12 and IL-23 cytokines by antibodies such as ustekinumab and brikianumab has been demonstrated to be effective in an experimental colitis model and in clinical trials in IBD patients, and in TNF- $\alpha$ antagonist-resistant IBD patients (65).

In addition to the use of antibodies for neutralizing proinflammatory cytokines, gene therapy with siRNA and oligonucleotide has recently investigated for treating inflammatory diseases. siRNA- mediated knockdown of pro-inflammatory cytokines has proved to be effective in reducing inflammatory conditions in various experimental models such as collageninduced arthritis, dextran sulfate-induced colitis (59). Among various siRNA downregulating pro-inflammatory cytokines, TNF- $\alpha$ siRNA-based therapy has been widely used for treating experimental models of RA and IBD. Electroporation of TNF$\alpha$ siRNA significantly reduced joint inflammation in murine collagen-induced arthritis (CIA) (66).

\section{Overexpression of Regulatory Cytokines}

Overexpression of regulatory cytokines is an alternative strategy for the treatment of inflammatory diseases. Among regulatory cytokines, IL-4 and IL-10, which are Th2 cell derive cytokines have received much attention for the treatment of inflammatory diseases in experimental models.
IL-4 and IL-10 have anti-inflammatory properties by suppressing the production of pro-inflammatory cytokines, chemokines such as TNF- $\alpha$ and IL-1 $\beta$, polarizing macrophage toward an anti-inflammatory M2 phenotype which counteracts inflammation via the release of IL-1Ra, IL-10 and TGF- $\beta$ and promote wound healing and tissue repair (67).

Plasmid DNA coding IL-10 gene has been a popular choice for overexpressing IL-10 cytokine in inflamed tissues of experimental IBD and RA. Our group has shown that murine IL-10 expressing plasmid DNA encapsulated in nanoparticles-inmicrospheres oral system (NiMOS) was effective in treating experimental colitis in mice (68). Likewise, treatment with plasmid DNA expressing IL-4 significantly reduced the incidence and severity of CIA (69). In addition, mouse IL-4 plasmid alone or in combination with mouse IL-10 plasmid have been used to prevent the development of autoimmune diabetes in non-obese diabetic mice, and inhibited trinitrobenzenesulfonic acid induced colon tissue damage (70).

\section{Delivery Challenges in Biological Therapy}

TNF- $\alpha$ has been a major target for monoclonal antibody therapy against inflammatory diseases with current FDA approval of infliximab, adalimumab, certolizumab pegzol, and golimumab for the treatment of RA and IBD (59). Blocking TNF- $\alpha$ receptors reduces inflammation and tissue damage in IBD and RA, but $40 \%$ of patients do not response to the current anti-TNF therapies (71). Furthermore, many patients experience symptomatic and functional impairment due toxicities related to the widespread distribution of the antibodies in solution dosage form and repeated high doses to achieve therapeutic concentrations (72). So far, formulations of the monoclonal antibodies are solutions administered via invasive systemic routes. The work by Lee et al. is among few studies that developed a targeted delivery system for a monoclonal antibody. A stable hyaluronate/gold nanoparticle/tocilizumab (HA-AuNP/TCZ) complex was synthesized for the successful treatment of RA in mouse model (73).

A gene therapy offers an innovative approach for treating inflammatory diseases due to its ability to achieve long-term expression of nucleic acids. However, unlike many proteins or peptides, nucleic acids have to enter cytoplasm or nucleus to be effective, resulting in significant delivery challenges. Unprotected nucleic acids have been reported to undergo rapid degradation in the circulation by the serum nucleases (74). In addition, following systemic administration, naked siRNA preferentially accumulated in kidney and removed by renal excretion due to its small size compared to glomerular pore size (75). Exogenous nucleic acids have been reported to induce innate immune responses via Toll-like receptors (75). Furthermore, the cellular uptake of nucleic acids is limited due to their high molecular weight, hydrophilic property, and negative charge (76). Once taken up by the cells, the endosome entrapment limits the release of nucleic acids to cytoplasm, which further reduces their activity. To overcome these challenges, various targeted delivery systems have been developed for biological therapies of inflammatory diseases that efficiently stabilize and deliver the nucleic acids into target cells. Targeted delivery systems allow specific delivery and sustained release of biologicals to inflamed tissues, hence enhancing their efficacy and reducing systemic toxicities. 


\section{MACROPHAGE REPOLARIZATION STRATEGY FOR INFLAMMATORY DISEASES}

\section{Macrophage Polarization Spectrum}

Macrophages are cells of the innate immune systems that play a number of crucial roles in inflammation, including phagocytosis, antigen presentation, and modulation of the immune response by releasing various cytokines and chemokines (77). Macrophages originate from bone marrow stem cells which develop into circulating monocytes, followed by regulated migration into different tissues where they differentiate into resident tissue macrophages (77). Tissueresident macrophages are able to rapid change their phenotype associated with morphologic, functional, and biochemical changes in response to local environment stimuli (78). Macrophages can be classified as "classically activated" or pro-inflammatory M1 phenotype, and "alternatively activated" or anti-inflammatory M2 phenotype (Fig. 4). The M1 phenotype is induced upon stimulation with IFN- $\gamma$ alone or in combination with bacterial lipopolysaccharide (LPS), while stimulation with IL-4/IL-13, and IL-10/TGF- $\beta$ results in the M2 phenotype (79). Each phenotype of macrophages shows typical expression profiles of surface markers as well as cytokines and chemokines. The M1 phenotype produces high level of pro-inflammatory cytokines such as TNF- $\alpha$, IL-1, IL6, IL-12, inducible nitric oxide synthase (iNOS), promotes Th1 and Th17 responses, and induces strong microbicidal and tumoricidal activity, thereby increasing inflammatory response. On the contrary, M2 macrophages produce high level of anti-inflammatory cytokines, such as IL-4 and IL-10, upregulate mannose receptors (CD206), arginase (Arg), and decrease the production of iNOS enzyme (80). M2 macrophages have immunosuppressive functions and are involved in pathogen clearance, alleviating inflammation, tissue repair and remodeling. Once polarized, M1 and M2 phenotype can switch the polarity upon exposure to the induction signals of opposite phenotype (81). This plasticity is a protective mechanism against infection or injury.

Upon infection or injury, circulating monocytes are recruited and transformed to M1 phenotype due to the presence of inflammatory stimuli in the injury tissue. In

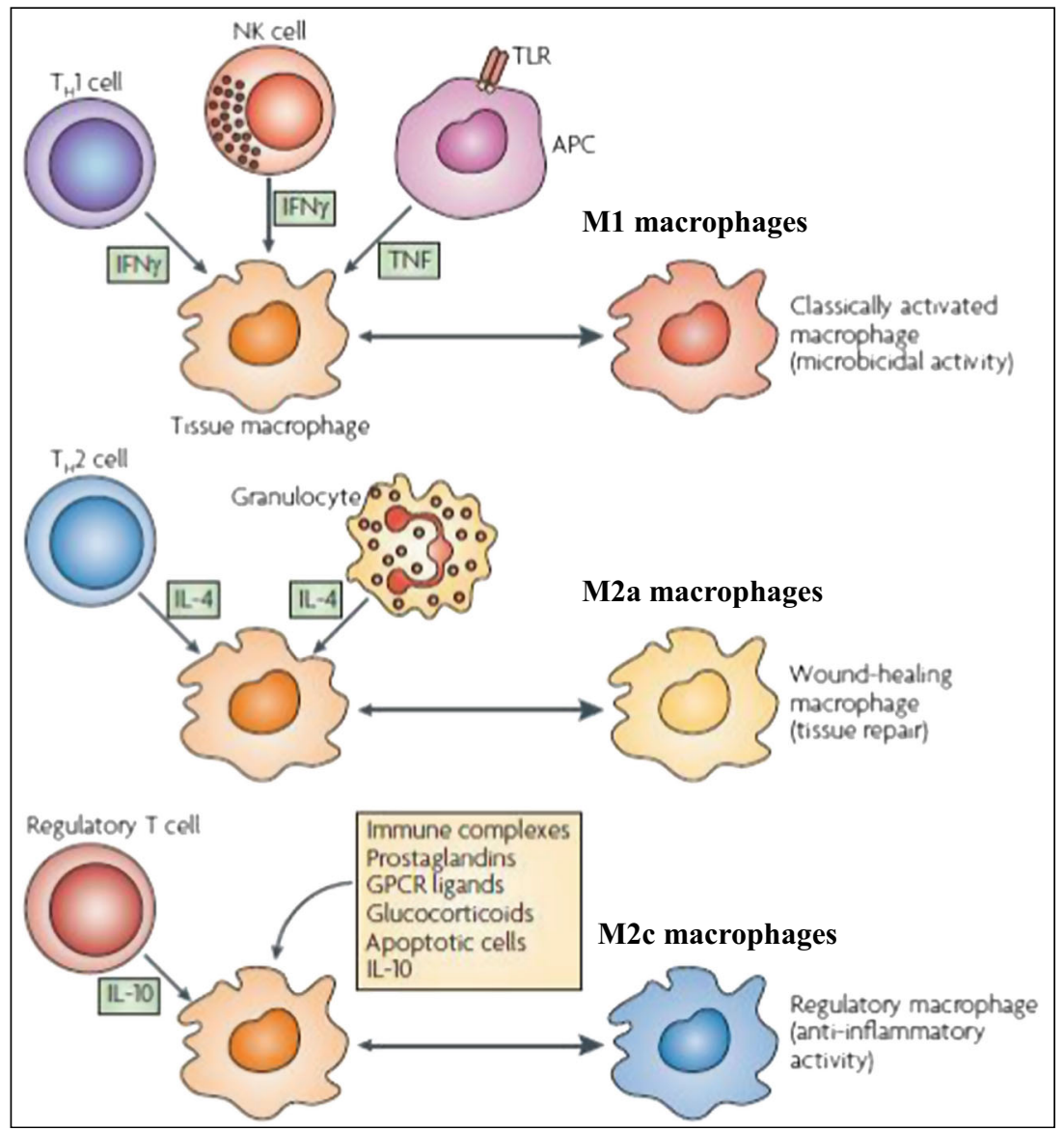

Fig. 4. Macrophage polarization upon exposure to different stimuli. M1 macrophages are induced in response to IFN- $\gamma$, which can be produced by Thelper 1 (TH1) cells or natural killer $(N K)$ cells, and tumor necrosis factor $(T N F)$, which is produced by antigen-presenting cells $(A P C s)$. M2a macrophages arise in response to interleukin-4 (IL-4), which can be produced by $\mathrm{TH} 2$ cells or granulocytes. M2c macrophages are generated in response to various stimuli, including immune complexes, prostaglandins, G-protein coupled receptor $(G P C R)$ ligands, glucocorticoids, apoptotic cells, or IL-10. TLR Toll-like receptor. Reprinted from Mosser and Edwards, 2008 with permission from NPG 
normal inflammation, the initial M1 phenotype changes to the anti-inflammatory M2 phenotype that serves to resolve the inflammation. However, in inflammatory diseases, the macrophage polarization is dysregulated and M1phenotype fail to return to M2 phenotypes (82). Therefore, modulation of macrophage polarization from an M1 to M2 state may be promising for the treatment of inflammatory diseases.

\section{M1-to-M2 Repolarization as an Anti-Inflammatory Therapeutic Strategy}

In recent years, emerging evidence has showed the involvement of various transcriptional pathways in macrophage polarization. Activation of $\mathrm{NF}-\kappa \mathrm{B}$, AP-1, CCAAT/ enhancer-binding protein $\alpha(\mathrm{C} / \mathrm{EBP}-\alpha)$, PU.1 and IFNregulatory factor 5 (IRF5) induced M1 activation, whereas stimulation of signal transducer and activator of transcription 6 (STAT6), PPAR- $\gamma$, IRF4, C/EBP- $\beta$, and Kruppel-like factor 4 (KLF4) polarized macrophages to the M2 phenotype (83). With insight into the transcriptional factors governing macrophage polarization and function, it is feasible to modulate macrophage phenotypes toward an anti-inflammatory phenotype. For example, IRF5 highly expressed in infarct macrophages during inflammation (M1) whereas its expression decreased during would healing process. silencing IRF5 by siRNA has been reported to suppress inflammatory M1 macrophages, supported resolution of inflammation, and infarct healing (84).

Recently, miRNAs, non-coding RNAs of 18-25 nucleotides, have emerged as novel regulators of immune responses in cancers, inflammatory, and autoimmune diseases. In addition, miRNAs have been reported to regulate macrophage polarization. Among various miRNAs, miR-124 has been reported to polarize macrophages toward the M2 phenotype (85). Overexpression of miR-124 in macrophages decreased expression of MHC class II and CD86 (M1 markers) and upregulation of Fizz1 and Arg1 (M2 markers). However, expression of miR-124 in microglia does not depend on IL-4 and/or IL-13 pathway, whereas miR-124 expression in lung macrophages was IL-4 and IL-13-dependent. Upregulation of several M2 markers and downregulation of the M1 markers in M2-polarized macrophages was absent by transfecting the macrophages with a miR-124 inhibitor $(85,86)$.

miR-223 is another miRNA that regulates M2 macrophage activation (87). Expression of miR-223 which highly enriched in bone marrow-derived macrophages and macrophages isolated from adipose tissue was dramatically increased in response to IL-4 but significantly decreased upon stimulation with LPS (88). Depletion of miR-223 in macrophages promotes M1 macrophage polarization and upregulates pro-inflammatory cytokines, such as TNF- $\alpha$, IL6 , and IL-1 $\beta$, which subsequently elevates the inflammation in adipose tissues. In contrast, the expression of Arg1, a marker for M2 macrophages, was diminished in miR-223-deficient macrophages (87). Likewise, Banerjee et al. reported that miR-let-7c and miR-125a-5p play important role in suppressing M1 macrophages while promoting M2 activation. These miRNAs are expressed at a higher level in M-BMM (M2 macrophages) than in GM-BMM (M1 macrophages). Overexpression of miR-let-7c and miR-125a-5p in GM-BMM diminished M1 phenotype induced by LPS but promoted M2 polarization by IL-4. In contrast, downregulation of miRlet-7c and miR-125a-5p in M-BMM promoted M1 polarization, and suppressed M2 phenotype expression. Furthermore, miR-let-7c and miR-125a-5p regulated bactericidal and phagocytic activities of macrophages. Increasing miR-let-7c and miR-125a-5p level diminished the bactericidal activity of macrophages while enhancing the phagocytic activity to ingest apoptotic cells. In contrast, knockdown of miR-let-7c and miR-125a-5p in M-BMM decreased their ability to phagocytosis $(89,90)$. Although, mainly focusing on cultured macrophages, these studies suggest that polarization of macrophages by gene therapy based on miR-223, miR-124, miR-125a-5p, and miR-let 7c may be a promising strategy for the treatment of inflammatory diseases.

\section{Macrophage-Targeted Delivery Systems}

Similar to cancers, inflamed tissues have leakage vasculature and overexpression of surface markers that can be exploited to engineer targeted delivery systems via passive targeting and active targeting. Vascular leakage is caused by the contraction of endothelial cells lining the arterioles and capillaries supplying the inflamed tissue due to the high expression of histamine, bradykinin, leukotrienes, and other mediators in the inflammatory state (91). Therefore, nanoparticles can extravasate into these interstitial spaces and be retained at the site of inflammation by the EPR effect (92).

Active targeting systems can be achieved by surface decoration of the nanoparticles with a targeting ligand that selectively interacts with target receptors on the surface of macrophages. Exploiting high expression of mannose and galactose receptors on the membrane of macrophages, various delivery systems have been modified with mannosecontaining polymers to achieve enhanced uptake in macrophages, especially in M2 macrophages where mannose receptors are upregulated (93). For example, mannosemodified trimethyl chitosan-cysteine (MTC) conjugate nanoparticles have been developed for targeting TNF- $\alpha$ to macrophages via oral delivery (94). Oral administration of MTC NPs containing low amount of TNF- $\alpha$ siRNA $(3.75 \mathrm{nM} /$ $\mathrm{kg}$ ) increased siRNA distribution to various organs including intestine and plasma compared to naked siRNA which inhibited systemic TNF- $\alpha$ production, and protected mice with acute hepatic injury from liver damage (94).

Dectin-1 receptor expressed in macrophages is essential for the phagocytosis of yeast through the recognition of $\beta$ glucans, a cellular component of yeast. Aouadi et al. have described the use of $\beta 1,3-D-g l u c a n-e n c a p s u l a t e d ~ s i R N A$ particles (GeRPs) generated from baker's yeast as efficient oral delivery vehicles to target $\beta$-glucan receptor overexpressed in inflamed macrophages (95). siRNA silencing TNF- $\alpha$ encapsulated in GeRPs showed extensive phagocytosis and efficient gene silencing in primary macrophages. GeRPs containing low dose of $20 \mu \mathrm{g} \mathrm{kg}$ siRNA was effective in silencing TNF- $\alpha$ in mouse macrophages in vivo following oral administration.

Folate receptor (FR) has been reported to express on the surface of M1 macrophages residing in the synovial fluid of RA which allows targeting these cells by folic acid-modified nanoparticles. Folate-conjugated chitosan has been designed 
for the delivery of plasmid DNA enconding IL-1R antagonist (IL-1Ra) in arthritic rats. Folate-targeted system significantly improved the transfection efficiency in FR-positive articular macrophages (96). Importantly, the targeted nanoparticles released IL-1Ra in a sustained manner in the serum for 10 days and significantly downregulated TNF- $\alpha$ and IL-1 $\beta$ providing protection against inflammatory bone resorption following intravenous administration.

In addition to actively targeting macrophages, targeting vascular endothelium may be useful for limitation of leukocyte recruitment and infiltration into the affected tissues in inflammatory. Leukocytes are recruited to the inflamed tissue by CAM containing three members including P-selectin, Eselectin and L-selectin (97). Kowalski et al. developed novel liposomes called SAINT-O-Somes for targeting siRNA to vascular cell adhesion molecule-1 (VCAM-1) or E-selectin which are overexpressed during inflammation (98). The liposomes modified with antibodies specific for E-selectin or VCAM-1 have shown higher release of siRNA in endothelial cells compared to conventional liposomes. Moreover, VEcadherin-specific siRNA encapsulated in SAINT-O-Somes can be successfully delivered into inflamed endothelial cells and significantly downregulated VCAM-1.

\section{Illustrative Examples of Macrophage Repolarization with Targeted Delivery}

IL-10 has been reported to polarize macrophages toward an anti-inflammatory M2 phenotype (67). Therefore, gene therapy based on plasmid DNA encoding IL-10 may be potential for M1-to-M2 macrophage repolarization.

Our group has developed NiMOS for delivery of plasmid DNA (pDNA) expressing IL-10 in a TNBS-induced acute colitis mice model. The NiMOS delivery system is composed of pDNA-encapsulated gelatin nanoparticles entrapped in a poly(epsilon-caprolactone) (PCL) microsphere. Since PCL is remained intact in the stomach but degraded by abundant lipases in the small and large intestine, NiMOS can protect DNA in the stomach and release the DNA-encapsulated nanoparticles specifically to the site of inflammation once the PCL matrix is degraded. The released plasmid DNAencapsulated gelatin nanoparticles can then be taken up by the enterocytes for delivery of the plasmid DNA. The overexpression of the IL-10 gene upon oral administration of NiMOS could successfully suppress the expression of proinflammatory cytokines in vivo including IFN- $\gamma$, TNF- $\alpha$, IL$1 \alpha$, IL-1 $\beta$, and IL-12, resulting in reversal of experimental colitis (68).

Our group has recently reported nanoparticles-inemulsions (NiE) system, which is comprised of gelatin nanoparticles encapsulated within a water-in-oil-in-water multiple emulsion, and tuftsin-modified alginate nanoparticles for macrophage-targeted delivery of plasmid DNA expressing mIL-10. J774A.1 cells treated with the NiE formulation or tuftsin-modified alginate nanoparticles containing plasmid DNA expressing mIL-10 showed higher expression of IL-10 compared to control nanoparticles or pDNA complexed with Lipofectamine. The overexpression of IL-10 by NiE or tuftsin-modified nanoparticles showed efficient antiinflammatory effects in J774A.1 cell by significantly reducing the levels of TNF- $\alpha$ and IL-1 $\beta$ produced in LPS-stimulated cells, indicating a promising strategy for the treatment of inflammatory diseases $(99,100)$.

Even though these studies showed overexpression of IL10 (M2 cytokine) which suppressed expression of TNF- $\alpha$ and IL-1 $\beta$ (M1 cytokines), more M1 and M2 markers such as surface markers need to be investigated to show full spectrum of M1-to-M2 macrophage repolarization by targeted delivery systems. So far, there has no targeted delivery system available for repolarization of macrophages toward antiinflammatory M2 phenotype by gene therapy.

\section{CONCLUSIONS}

Despite the advances in the availability of therapies for resistant cancers and inflammatory diseases, there is a significant need to facilitate clinical translation of advances in molecular medicine into effective disease diagnosis and therapeutic strategies. Nanotechnology has an important role to play in disease diagnosis, imaging, and therapy and potentially may advance personalized medicine. Several targeting strategies have been adopted to modify these nanoparticles for anticancer or anti-inflammatory therapy. The focus of these delivery systems is on solving important medical problems with innovative solutions that use inexpensive and safe materials, as well as, scalable fabrication methods so that these promising experimental technologies are realized in the clinic in the near future.

\section{ACKNOWLEDGMENTS}

Our work on development of nanoparticulate delivery systems for the treatment of refractory cancer has been generously supported by the National Cancer Institute's (NCI) Cancer Nanotechnology Platform Partnership (CNPP) grants from 2005 to 2015 and the Dana Farber Cancer InstituteNortheastern University Collaborative Grant on Development of Cancer Therapeutics. The authors would also like to acknowledge Dr. Lara Milane, Dr. Arun Iyer, Dr. Shanthi Ganesh, Dr. Amit Singh, and Dr. Shardool Jain for their valuable contributions to the projects discussed in this review.

\section{REFERENCES}

1. Siegel R, Ma J, Zou Z, Jemal A. Cancer statistics, 2014. CA Cancer J Clin. 2014;64(1):9-29.

2. Dougan M, Dranoff G. Immunotherapy of cancer. Innate immune regulation and cancer immunotherapy: Springer; 2012. p. 391-414.

3. Cowen R, Garside E, Fitzpatrick B, Papadopoulou M, Williams $\mathrm{K}$. Gene therapy approaches to enhance bioreductive drug treatment. 2014.

4. Neurath MF. Cytokines in inflammatory bowel disease. Nat Rev Immunol. 2014;14(5):329-42.

5. Wirtz S, Neurath MF. Gene transfer approaches for the treatment of inflammatory bowel disease. Gene Ther. 2003;10(10):854-60.

6. Aznavoorian S, Stracke ML, Krutzsch H, Schiffmann E, Liotta LA. Signal transduction for chemotaxis and haptotaxis by matrix molecules in tumor cells. J Cell Biol. 1990;110(4):1427-38.

7. Ohtani H. Stromal reaction in cancer tissue: pathophysiologic significance of the expression of matrix-degrading enzymes in 
relation to matrix turnover and immune/inflammatory reactions. Pathol Int. 1998;48(1):1-9.

8. Jain RK, Martin JD, Stylianopoulos T. The role of mechanical forces in tumor growth and therapy. Annu Rev Biomed Eng. 2014;16:321.

9. Carmeliet P, Jain RK. Angiogenesis in cancer and other diseases. Nature. 2000;407(6801):249-57.

10. Gottesman MM. Mechanisms of cancer drug resistance. Annu Rev Med. 2002;53(1):615-27.

11. Copeland RA, Pompliano DL, Meek TD. Drug-target residence time and its implications for lead optimization. Nat Rev Drug Discov. 2006;5(9):730-9.

12. Fattal E, Bochot A. Ocular delivery of nucleic acids: antisense oligonucleotides, aptamers and siRNA. Adv Drug Deliv Rev. 2006;58(11):1203-23.

13. Fujita Y, Takeshita F, Kuwano K, Ochiya T. RNAi therapeutic platforms for lung diseases. Pharmaceuticals. 2013;6(2):223-50.

14. Rao J. Shedding light on tumors using nanoparticles. ACS Nano. 2008;2(10):1984-6.

15. Talekar M, Ganta S, Singh A, Amiji M, Kendall J, Denny WA, et al. Phosphatidylinositol 3-kinase inhibitor (PIK75) containing surface functionalized nanoemulsion for enhanced drug delivery, cytotoxicity and pro-apoptotic activity in ovarian cancer cells. Pharm Res. 2012;29(10):2874-86.

16. Talekar M, Kendall J, Denny W, Garg S. Targeting of nanoparticles in cancer: drug delivery and diagnostics. AntiCancer Drugs. 2011;22(10):949-62.

17. Wang S-N, Deng Y-H, Xu H, Wu H-B, Qiu Y-K, Chen D-W. Synthesis of a novel galactosylated lipid and its application to the hepatocyte-selective targeting of liposomal doxorubicin. Eur J Pharm Biopharm. 2006;62(1):32-8.

18. Kim D, Jeong YY, Jon S. A drug-loaded aptamer-gold nanoparticle bioconjugate for combined CT imaging and therapy of prostate cancer. ACS Nano. 2010;4(7):3689-96.

19. Mulik RS, Mönkkönen J, Juvonen RO, Mahadik KR, Paradkar AR. Transferrin mediated solid lipid nanoparticles containing curcumin: enhanced in vitro anticancer activity by induction of apoptosis. Int J Pharm. 2010;398(1-2):190-203.

20. Sun B, Ranganathan B, Feng S-S. Multifunctional poly (D, Llactide-co-glycolide)/montmorillonite (PLGA/MMT) nanoparticles decorated by Trastuzumab for targeted chemotherapy of breast cancer. Biomaterials. 2008;29(4):475-86.

21. Ito A, Kuga Y, Honda H, Kikkawa H, Horiuchi A, Watanabe Y, et al. Magnetite nanoparticle-loaded anti-HER2 immunoliposomes for combination of antibody therapy with hyperthermia. Cancer Lett. 2004:212(2):167-75.

22. Xu J AM. Therapeutic gene delivery and transfection in human pancreatic cancer cells using epidermal growth factor receptortargeted gelatin nanoparticles. J Vis Exp. 2012;4(59)

23. Xu JGF, Amiji M. Biodistribution and pharmacokinetics of EGFR-targeted thiolated gelatin nanoparticles following systemic administration in pancreatic tumor-bearing mice. Mol Pharm. 2013;10(5):2031-44.

24. Milane L, Duan ZF, Amiji M. Pharmacokinetics and biodistribution of lonidamine/paclitaxel loaded, EGFRtargeted nanoparticles in an orthotopic animal model of multi-drug resistant breast cancer. Nanomedicine: NBM. 2011;7(4):435-44.

25. Bertout JA, Patel SA, Simon MC. The impact of $\mathrm{O}_{2}$ availability on human cancer. Nat Rev Cancer. 2008;8(12):967-75.

26. Wilson WR, Hay MP. Targeting hypoxia in cancer therapy. Nat Rev Cancer. 2011;11(6):393-410.

27. Shannon AM, Bouchier-Hayes DJ, Condron CM, Toomey D. Tumour hypoxia, chemotherapeutic resistance and hypoxiarelated therapies. Cancer Treat Rev. 2003;29(4):297-307.

28. Yamasaki M, Nomura T, Sato F, Mimata H. Metallothionein is up-regulated under hypoxia and promotes the survival of human prostate cancer cells. Oncol Rep. 2007;18(5):1145-53.

29. Greer SN, Metcalf JL, Wang Y, Ohh M. The updated biology of hypoxia-inducible factor. EMBO J. 2012;31(11):2448-60.

30. Rankin EB, Giaccia AJ. The role of hypoxia-inducible factors in tumorigenesis. Cell Death Differ. 2008;15(4):678-85.

31. Powis G, Kirkpatrick L. Hypoxia inducible factor- $1 \alpha$ as a cancer drug target. Mol Cancer Ther. 2004;3(5):647-54.
32. Gatenby RA, Gawlinski ET, Gmitro AF, Kaylor B, Gillies RJ. Acid-mediated tumor invasion: a multidisciplinary study. Cancer Res. 2006;66(10):5216-23.

33. Ganta S, Devalapally H, Shahiwala A, Amiji M. A review of stimuli-responsive nanocarriers for drug and gene delivery. $\mathrm{J}$ Control Release. 2008;126(3):187-204.

34. Thambi T, Deepagan VG, Yoon HY, Han HS, Kim SH, Son S, et al. Hypoxia-responsive polymeric nanoparticles for tumortargeted drug delivery. Biomaterials. 2014;35(5):1735-43.

35. Poon Z, Chang D, Zhao X, Hammond PT. Layer-by-layer nanoparticles with a $\mathrm{pH}$-sheddable layer for in vivo targeting of tumor hypoxia. ACS Nano. 2011;5(6):4284-92.

36. Liu XQ, Xiong MH, Shu XT, Tang RZ, Wang J. Therapeutic delivery of siRNA silencing HIF-1 alpha with micellar nanoparticles inhibits hypoxic tumor growth. Mol Pharm. 2012;9(10):2863-74.

37. Jiang X, Xin H, Ren Q, Gu J, Zhu L, Du F, et al. Nanoparticles of 2-deoxy-D-glucose functionalized poly(ethylene glycol)-copoly(trimethylene carbonate) for dual-targeted drug delivery in glioma treatment. Biomaterials. 2014;35(1):518-29.

38. Pathania D, Millard M, Neamati N. Opportunities in discovery and delivery of anticancer drugs targeting mitochondria and cancer cell metabolism. Adv Drug Deliv Rev. 2009;61(14):125075.

39. Milane L, Duan Z, Amiji M. Therapeutic efficacy and safety of paclitaxel/lonidamine loaded EGFR-targeted nanoparticles for the treatment of multi-drug resistant cancer. PLoS One. 2011;6(9), e24075.

40. Guo W, Zhang Y, Chen T, Wang Y, Xue J, Zhang Y, et al. Efficacy of RNAi targeting of pyruvate kinase M2 combined with cisplatin in a lung cancer model. J Cancer Res Clin Oncol. 2011;137(1):65-72.

41. Shi HS, Li D, Zhang J, Wang YS, Yang L, Zhang HL, et al. Silencing of PKM-2 increases the efficacy of docetaxel in human lung cancer xenografts in mice. Cancer Sci. 2010;101(6):144753.

42. Goldberg MS, Sharp PA. Pyruvate kinase M2-specific siRNA induces apoptosis and tumor regression. J Exp Med. 2012;209(2):217-24.

43. Yadav S, van Vlerken LE, Little SR, Amiji MM. Evaluations of combination MDR-1 gene silencing and paclitaxel administration in biodegradable polymeric nanoparticle formulations to overcome multidrug resistance in cancer cells. Cancer Chemother Pharmacol. 2009;63(4):711-22.

44. Kobayashi E, Iyer AK, Hornicek FJ, Amiji MM, Duan Z. Lipid-functionalized dextran nanosystems to overcome multidrug resistance in cancer: a pilot study. Clin Orthop Relat Res. 2013;471(3):915-25.

45. Ganesh S, Iyer AK, Gattacceca F, Morrissey DV, Amiji MM. In vivo biodistribution of siRNA and cisplatin administered using CD44-targeted hyaluronic acid nanoparticles. J Control Release. 2013;172(3):699-706.

46. Ganesh S, Iyer AK, Morrissey DV, Amiji MM. Hyaluronic acid based self-assembling nanosystems for CD44 target mediated siRNA delivery to solid tumors. Biomaterials. 2013;34(13)):3489-502.

47. Pan B, Cui D, Sheng Y, Ozkan C, Gao F, He R, et al. Dendrimer-modified magnetic nanoparticles enhance efficiency of gene delivery system. Cancer Res. 2007;67(17):8156-63.

48. van Vlerken L, Shenoy D, Duan Z, Selden M, Mehta S, Amiji $\mathrm{M}$, editors. Modulation of intracellular ceramide using polymeric nanoparticles to overcome multidrug resistance in tumor cells. Annual Meeting-Society For Biomaterials In Conjunction With The International Biomaterials Symposium; 2006.

49. Yu C, Zhang X, Sun G, Guo X, Li H, You Y, et al. RNA interference-mediated silencing of the polo-like kinase 1 gene enhances chemosensitivity to gemcitabine in pancreatic adenocarcinoma cells. J Cell Mol Med. 2008;12(6a):2334-49.

50. Reagan-Shaw S, Ahmad N. Silencing of polo-like kinase (Plk) 1 via siRNA causes induction of apoptosis and impairment of mitosis machinery in human prostate cancer cells: implications for the treatment of prostate cancer. FASEB J. 2005;19(6):6113.

51. Bu Y, Yang Z, Li Q, Song F. Silencing of polo-like kinase (Plk) 1 via siRNA causes inhibition of growth and induction of 
apoptosis in human esophageal cancer cells. Oncology. 2008;74(3):198.

52. Nascimento AV, Singh A, Bousbaa H, Ferreira D, Sarmento B, Amiji MM. Mad2 checkpoint gene silencing using epidermal growth factor receptor-targeted chitosan nanoparticles in nonsmall cell lung cancer model. Mol Pharm. 2014;11(10):3515-27.

53. Abeylath SC, Amiji MM. 'Click'synthesis of dextran macrostructures for combinatorial-designed self-assembled nanoparticles encapsulating diverse anticancer therapeutics. Bioorg Med Chem. 2011;19(21):6167-73.

54. Lynn DM, Anderson DG, Putnam D, Langer R. Accelerated discovery of synthetic transfection vectors: parallel synthesis and screening of a degradable polymer library. J Am Chem Soc. 2001;123(33):8155-6.

55. Akinc A, Anderson DG, Lynn DM, Langer R. Synthesis of poly ( $\beta$-amino ester) s optimized for highly effective gene delivery. Bioconjug Chem. 2003;14(5):979-88.

56. Greenland JR, Liu H, Berry D, Anderson DG, Kim WK, Irvine $\mathrm{DJ}$, et al. $\beta$-amino ester polymers facilitate in vivo DNA transfection and adjuvant plasmid DNA immunization. Mol Ther. 2005;12(1):164-70.

57. Tabas I, Glass CK. Anti-inflammatory therapy in chronic disease: challenges and opportunities. Science. 2013;339(6116):166-72.

58. Sousa LP, Alessandri AL, Pinho V, Teixeira MM. Pharmacological strategies to resolve acute inflammation. Curr Opin Pharmacol. 2013;13(4):625-31.

59. Tran TH, Amiji MM. Targeted delivery systems for biological therapies of inflammatory diseases. Expert Opin Drug Deliv. 2014;4:1-22.

60. Dinarello CA. Anti-inflammatory agents: present and future. Cell. 2010;140(6):935-50.

61. Van den Brande JM, Braat H, van den Brink GR, Versteeg $\mathrm{HH}$, Bauer CA, Hoedemaeker I, et al. Infliximab but not etanercept induces apoptosis in lamina propria T-lymphocytes from patients with Crohn's disease. Gastroenterology. 2003;124(7):1774-85.

62. Ding C, Cicuttini F, Li J, Jones G. Targeting IL-6 in the treatment of inflammatory and autoimmune diseases. Expert Opin Investig Drugs. 2009;18(10):1457-66.

63. Nishimoto N, Miyasaka N, Yamamoto K, Kawai S, Takeuchi T, Azuma J, et al. Study of active controlled tocilizumab monotherapy for rheumatoid arthritis patients with an inadequate response to methotrexate (SATORI): significant reduction in disease activity and serum vascular endothelial growth factor by IL-6 receptor inhibition therapy. Mod Rheumatol. 2009;19(1):12-9.

64. Burmester GR, Feist E, Dorner T. Emerging cell and cytokine targets in rheumatoid arthritis. Nat Rev Rheumatol. 2014;10(2):77-88.

65. Sandborn WJ, Gasink C, Gao LL, Blank MA, Johanns J, Guzzo $\mathrm{C}$, et al. Ustekinumab induction and maintenance therapy in refractory Crohn's disease. N Engl J Med. 2012;367(16):151928.

66. Schiffelers RM, Xu J, Storm G, Woodle MC, Scaria PV. Effects of treatment with small interfering RNA on joint inflammation in mice with collagen-induced arthritis. Arthritis Rheum. 2005;52(4):1314-8.

67. Gordon S. Alternative activation of macrophages. Nat Rev Immunol. 2003;3(1):23-35.

68. Bhavsar MD, Amiji MM. Oral IL-10 gene delivery in a microsphere-based formulation for local transfection and therapeutic efficacy in inflammatory bowel disease. Gene Ther. 2008;15(17):1200-9.

69. Kageyama Y, Koide Y, Uchijima M, Nagata T, Yoshida A, Taiki A, et al. Plasmid encoding interleukin-4 in the amelioration of murine collagen-induced arthritis. Arthritis Rheum. 2004;50(3):968-75.

70. Xiong J, Lin YH, Bi LH, Wang JD, Bai Y, Liu SD. Effects of interleukin-4 or interleukin-10 gene therapy on trinitrobenzenesulfonic acid-induced murine colitis. BMC Gastroenterol. 2013;13:165.

71. Khoury M, Escriou V, Courties G, Galy A, Yao R, Largeau C, et al. Efficient suppression of murine arthritis by combined anticytokine small interfering RNA lipoplexes. Arthritis Rheum. 2008;58(8):2356-67.

72. Yuan F, Quan LD, Cui L, Goldring SR, Wang D. Development of macromolecular prodrug for rheumatoid arthritis. Adv Drug Deliv Rev. 2012;64(12):1205-19.

73. Lee H, Lee MY, Bhang SH, Kim BS, Kim YS, Ju JH, et al. Hyaluronate-gold nanoparticle/tocilizumab complex for the treatment of rheumatoid arthritis. ACS Nano. 2014;8(5):4790-8.

74. Bumcrot D, Manoharan M, Koteliansky V, Sah DW. RNAi therapeutics: a potential new class of pharmaceutical drugs. Nat Chem Biol. 2006;2(12):711-9.

75. Deng Y, Wang CC, Choy KW, Du Q, Chen J, Wang Q, et al. Therapeutic potentials of gene silencing by RNA interference: principles, challenges, and new strategies. Gene. 2014;538(2):217-27.

76. Aagaard L, Rossi JJ. RNAi therapeutics: principles, prospects and challenges. Adv Drug Deliv Rev. 2007;59(2-3):75-86.

77. Gordon S, Taylor PR. Monocyte and macrophage heterogeneity. Nat Rev Immunol. 2005;5(12):953-64.

78. Mosser DM, Edwards JP. Exploring the full spectrum of macrophage activation. Nat Rev Immunol. 2008;8(12):95869.

79. Hamilton TA, Ohmori Y, Tebo J. Regulation of chemokine expression by antiinflammatory cytokines. Immunol Res. 2002;25(3):229-45.

80. Katakura T, Miyazaki M, Kobayashi M, Herndon DN, Suzuki F. CCL17 and IL-10 as effectors that enable alternatively activated macrophages to inhibit the generation of classically activated macrophages. J Immunol. 2004;172(3):1407-13.

81. Stout RD, Jiang C, Matta B, Tietzel I, Watkins SK, Suttles J. Macrophages sequentially change their functional phenotype in response to changes in microenvironmental influences. J Immunol. 2005;175(1):342-9.

82. Nathan C, Ding A. Nonresolving inflammation. Cell. 2010;140(6):871-82.

83. Lawrence T, Natoli G. Transcriptional regulation of macrophage polarization: enabling diversity with identity. Nat Rev Immunol. 2011;11(11):750-61.

84. Courties G, Heidt T, Sebas M, Iwamoto Y, Jeon D, Truelove J, et al. In vivo silencing of the transcription factor IRF5 reprograms the macrophage phenotype and improves infarct healing. J Am Coll Cardiol. 2014;63(15):1556-66.

85. Veremeyko T, Siddiqui S, Sotnikov I, Yung A, Ponomarev ED. IL-4/IL-13-dependent and independent expression of miR-124 and its contribution to M2 phenotype of monocytic cells in normal conditions and during allergic inflammation. PLoS One. 2013;8(12), e81774.

86. Ponomarev ED, Veremeyko T, Barteneva N, Krichevsky AM, Weiner HL. MicroRNA-124 promotes microglia quiescence and suppresses EAE by deactivating macrophages via the C/EBPalpha-PU.1 pathway. Nat Med. 2011;17(1):64-70.

87. Zhuang G, Meng C, Guo X, Cheruku PS, Shi L, Xu H, et al. A novel regulator of macrophage activation: miR-223 in obesityassociated adipose tissue inflammation. Circulation. 2012;125(23):2892-903.

88. Fazi F, Rosa A, Fatica A, Gelmetti V, De Marchis ML, Nervi C, et al. A minicircuitry comprised of microRNA-223 and transcription factors NFI-A and C/EBPalpha regulates human granulopoiesis. Cell. 2005;123(5):819-31.

89. Banerjee $\mathrm{S}$, Xie N, Cui H, Tan Z, Yang S, Icyuz M, et al. MicroRNA let-7c regulates macrophage polarization. J Immunol. 2013;190(12):6542-9.

90. Banerjee S, Cui H, Xie N, Tan Z, Yang S, Icyuz M, et al. miR$125 \mathrm{a}-5 \mathrm{p}$ regulates differential activation of macrophages and inflammation. J Biol Chem. 2013;288(49):35428-36.

91. Lampugnani MG, Dejana E. Interendothelial junctions: structure, signalling and functional roles. Curr Opin Cell Biol. 1997;9(5):674-82.

92. Maeda H, Wu J, Sawa T, Matsumura Y, Hori K. Tumor vascular permeability and the EPR effect in macromolecular therapeutics: a review. J Control Release. 2000;65(1-2):271-84.

93. Wattendorf U, Coullerez G, Voros J, Textor M, Merkle HP. Mannose-based molecular patterns on stealth microspheres for 
receptor-specific targeting of human antigen-presenting cells. Langmuir. 2008;24(20):11790-802.

94. He C, Yin L, Tang C, Yin C. Multifunctional polymeric nanoparticles for oral delivery of TNF-alpha siRNA to macrophages. Biomaterials. 2013;34(11):2843-54.

95. Aouadi M, Tesz GJ, Nicoloro SM, Wang M, Chouinard M, Soto E, et al. Orally delivered siRNA targeting macrophage Map4k4 suppresses systemic inflammation. Nature. 2009;458(7242):1180-4.

96. Shi Q, Wang H, Tran C, Qiu X, Winnik FM, Zhang X, et al. Hydrodynamic delivery of chitosan-folate-DNA nanoparticles in rats with adjuvant-induced arthritis. J Biomed Biotechnol. 2011;2011:148763.

97. Ulbrich H, Eriksson EE, Lindbom L. Leukocyte and endothelial cell adhesion molecules as targets for therapeutic interventions in inflammatory disease. Trends Pharmacol Sci. 2003;24(12):640-7.

98. Kim I, Moon SO, Kim SH, Kim HJ, Koh YS, Koh GY. Vascular endothelial growth factor expression of intercellular adhesion molecule 1 (ICAM-1), vascular cell adhesion molecule 1 (VCAM-1), and E-selectin through nuclear factor-kappa B activation in endothelial cells. J Biol Chem. 2001;276(10):761420.

99. Attarwala H, Amiji M. Multi-compartmental nanoparticles-inemulsion formulation for macrophage-specific anti-inflammatory gene delivery. Pharm Res. 2012;29(6):1637-49.

100. Jain S, Amiji M. Tuftsin-modified alginate nanoparticles as a noncondensing macrophage-targeted DNA delivery system. Biomacromolecules. 2012;13(4):1074-85. 